\title{
The complementation of yeast with human or Plasmodium falciparum Hsp90 confers differential inhibitor sensitivities
}

\author{
Diana Wider ${ }^{\mathrm{a}}$, Marie-Pierre Péli-Gulli ${ }^{\mathrm{a}, 1}$, Pierre-André Briand ${ }^{\mathrm{a}}$, Utpal Tatu ${ }^{\mathrm{b}}$, Didier Picard ${ }^{\mathrm{a}, *}$ \\ a Département de Biologie Cellulaire, Université de Genève, Sciences III, 30 quai Ernest-Ansermet, CH-1211 Genève 4, Switzerland \\ ${ }^{\mathrm{b}}$ Department of Biochemistry, Indian Institute of Science, Bangalore 560012, India
}

\begin{abstract}
Developing novel drugs against the unicellular parasite Plasmodium is complicated by the paucity of simple screening systems. Heat-shock proteins are an essential class of proteins for the parasite's cyclical life style between different cellular milieus and temperatures. The molecular chaperone Hsp90 assists a large variety of proteins, but its supporting functions for many proteins that are important for cancer have made it into a well-studied drug target. With a better understanding of the differences between Hsp90 of the malarial parasite and Hsp90 of its human host, new therapeutic options might become available. We have generated a set of isogenic strains of the budding yeast Saccharomyces cerevisiae where the essential yeast Hsp90 proteins have been replaced with either of the two human cytosolic isoforms Hsp90 $\alpha$ or Hsp90 3 , or with Hsp90 from Plasmodium falciparum (Pf). All strains express large amounts of the Flagtagged Hsp90 proteins and are viable. Even though the strain with Pf Hsp90 grows more poorly, it provides a tool to reconstitute additional aspects of the parasite Hsp90 complex and its interactions with substrates in yeast as a living test tube. Upon exposure of the set of Hsp90 test strains to the two Hsp90 inhibitors radicicol (Rd) and geldanamycin (GA), we found that the strain with Pf Hsp90 is relatively more sensitive to GA than to Rd compared to the strains with human Hsp90's. This indicates that this set of yeast strains could be used to screen for new Pf Hsp90 inhibitors with a wider therapeutic window.
\end{abstract}

\section{Introduction}

WHO estimates that over 3 billion people live under the threat of malaria. The malarial parasite Plasmodium infects over 500 million people annually, which results in 3 million deaths. Discovering and developing new inhibitors, for example for Plasmodium falciparum (Pf), is difficult as screening depends on the costly and laborious maintenance of parasite cultures and on determining parasitemia in rodent models. New molecular targets and experimental systems would be a welcome addition to the toolbox. Since unicellular parasites are exposed to extreme changes in their environment as they cycle between host and vector, their heat-shock proteins may play an important role. The molecular chaperone Hsp90 has been shown to be essential for viability in organisms ranging from yeast to mouse [1-3]. The life cycle of Pf has been shown to be blocked in vitro with two chemically distinct Hsp90 inhibitors, radicicol (Rd) and geldanamycin (GA), primarily at the transition from the Ring to the trophozoite stage in erythrocytes [4-7]. The stage-specificity of

\footnotetext{
* Corresponding author. Tel.: +41 22379 6813; fax: +41223796928.

E-mail address: didier.picard@unige.ch (D. Picard).

1 Present address: Département de Médecine, Unité de Biochimie, Université de Fribourg, CH-1700 Fribourg, Switzerland.
}

the inhibition has been taken as an indication that the drugs might inhibit primarily through the parasite Hsp90.

Because Hsp90 has been recognized as an Achilles heel of cancer cells, there are already a considerable number of drugs available and more are being developed, and clinical trials are currently being conducted with GA derivatives [2,8,9]. These studies have provided a lot of information on the pharmacology of such inhibitors, and so far, they have revealed surprisingly few side effects. In addition, a recent study showed that Hsp90 inhibitors block infection of tissue culture cells by a wide panel of different viruses [10]. Not surprisingly, it has been suggested that the already available Hsp90 inhibitors could be further developed as anti-viral and anti-parasite agents. Hsp90 is highly conserved as illustrated by the fact that GA binds and inhibits both Pf and human Hsp90, and yet, within the constraints of $63 \%$ sequence identity at the amino acid level, there should be sufficient sequence and structural differences to identify inhibitors that are selective for the parasite Hsp90 [11].

The budding yeast Saccharomyces cerevisiae (S. cerevisiae) was the first organism for which it was shown that Hsp90 is essential for viability [12]. This genetically easily manipulable organism has two genes, HSC82 and HSP82, which encode two 95\% identical Hsp90 proteins. Interestingly, heterologous Hsp90 proteins from many species are able to replace Hsp82 and Hsc82 of the budding yeast. These include the two human cytosolic isoforms Hsp90 $\alpha$ [13] and 
Hsp90ß [14], Hsp90 from Trypanosoma cruzi [15], Drosophila [16], Caenorhabditis elegans and Candida albicans [17]. S. cerevisiae lends itself perfectly to explore the functional conservation of proteins from other species, to study their homologous and heterologous biochemical interactions, and to screen for novel species-specific drugs. This provides the rationale for why we decided to generate and to study an isogenic set of yeast strains with human and Pf Hsp90.

\section{Materials and methods}

\subsection{Plasmids}

The backbone for all constructs is plasmid pRS313/GPD-PGK (a gift from A. Kralli). It is derived from the low copy number CEN/ARS yeast expression vector pRS313 [18] by insertion of the strong constitutive GPD promoter from the TDH3 gene and the PGK1 terminator. Plasmid pHCA/hHsp90 $\beta$ for expression of untagged human Hsp90 $\beta$ was derived from p2TG/hHsp90 $\beta$ [19] and has been described before [20]. Plasmid pRS313/PfHsp90 for expression of untagged Pf Hsp90 was made by transferring the Hsp90 open reading frame (ORF) from plasmid pGEX-PfHsp90 as a PCR fragment containing the sequence GGATCCAAAAA and an EcoRI site preceding and following the ORF, respectively. For expression of Flag-tagged Hsp90 proteins, the expression vector pHGF was derived from plasmid pRS313/GPDPGK by replacing the multiple cloning sequences encompassing the BamHI and HindIII sites with the sequence GGATCGCCACCATGGATTACAAAGATGATGATGATAAAGGGATCCAAGCTT (sequences encoding initiator methionine and Flag tag are underlined). Hsp90 ORFs were inserted into the BamHI or HindIII sites of pHGF to generate plasmids pHGF/Hsp82, pHGF/PfHsp90, pHGF/HsHsp90 $\alpha$ and pHGF/HsHsp90 $\beta$ to express Flag-tagged yeast Hsp82 (one of the two yeast Hsp90 isoforms), Pf Hsp90, human Hsp90 $\alpha$ and Hsp90 $\beta$, respectively.

\subsection{Yeast strains}

All strains are derived from strain pp30\#10 [21], which has the following genotype: MATa ade2-101-o his3- $\Delta 200$ leu2-3,112 lys2-801-a trp1-289 ura3-52 $\Delta$ hsc82::KanMx4 $\Delta$ hsp82::KanMx4 / $2 \mu$-HSC82-URA3. It carries deletions of the two yeast HSP90 genes HSP82 and HSC82 and is kept alive by expression of Hsc82 from an episome. To sensitize the strain for Hsp90 inhibitors [22], we deleted the gene encoding the export pump Pdr5 by using homologous recombination to replace the coding region with the loxP-LEU2-loxP cassette from plasmid pUG73 [23]. This generated yeast strain DP533. All others were derived from DP533 by plasmid shuffling. Specifically, strains DP549, DP551, DP552, DP553, DP554, and DP555, referred herein also as $\mathrm{P}, \beta, \mathrm{FY}, \mathrm{FP}, \mathrm{F} \beta$, and $\mathrm{F} \alpha$, contain plasmids pRS313/PfHsp90, pHCA/hHsp90 $\beta$, pHGF/Hsp82, pHGF/PfHsp90, pHGF/HsHsp90 $\beta$, and pHGF/HsHsp90 $\alpha$, respectively. The strains with human Hsp90's being genetically highly similar, their correctness could also be double-checked with isoform-specific antibodies (data not shown, see also ref. [20]).

\subsection{Preparation of yeast extracts and immunoblotting}

Cells were grown to an OD600 of 0.7-0.8 in rich medium with $2 \%$ glucose and $200 \mu \mathrm{g} / \mathrm{ml}$ adenine (YAPD), collected and washed with ice-cold water. Cell pellets were resuspended in $10 \mathrm{mM}$ Tris- $\mathrm{HCl} \mathrm{pH} 7.5,50 \mathrm{mM} \mathrm{NaCl}, 10 \mathrm{mM} \mathrm{MgCl}_{2}, 1 \mathrm{mM}$ EDTA, $1 \mathrm{mM}$ DTT, $20 \%(\mathrm{v} / \mathrm{v})$ glycerol, and a cocktail of protease inhibitors. Cells were broken mechanically with glass beads, and cleared lysates

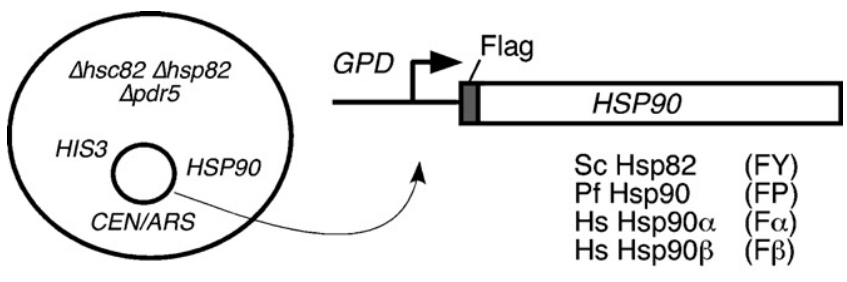

Fig. 1. Schematic representation of the isogenic set of Hsp90 tester strains. The species are abbreviated as follows: Sc, Saccharomyces cerevisiae; Pf, Plasmodium falciparum; Hs, Homo sapiens. The dotted line below the list of Hsp90 proteins indicates that this system can easily accommodate Hsp90 proteins from other species. Further details are given in the text.

quantitated to load equal amounts of proteins onto denaturing SDSpolyacrylamide gels. The immunoblots were processed as described before [20].

\subsection{Growth assays}

YAPD medium was used both for plates and for liquid cultures and cells were grown at $30^{\circ} \mathrm{C}$. Cells were freshly grown from an overnight inoculum to OD600 $\sim 0.5$ before starting the assays. For plate assays, $5 \mu \mathrm{l}$ aliquots of a serial dilution series were spotted, starting with a dilution to OD600 $=0.05$. Plates were scanned after 3 and 4 days. For assays with liquid cultures, triplicate samples of cells were diluted to OD600 $=0.05$ except for FY, which was diluted further to 0.01. Cell densities were measured at appropriate dilutions with a spectrophotometer at OD600. Where indicated, Rd and GA were added from $10 \mathrm{mM}$ stocks in dimethyl sulfoxide (DMSO). The untreated control plates and cultures received the same amount of DMSO (vehicle) needed as a solvent for the samples with the highest drug concentration.

\section{Results}

We used plasmid shuffling to replace the endogenous HSP9O genes of S. cerevisiae, HSC82 and HSP82, with HSP90 from different species. The latter were expressed from a low copy plasmid under the control of the strong constitutive GPD promoter. To facilitate the comparison of Hsp90 protein levels, we also generated a set of strains expressing all Hsp90 proteins with an N-terminal Flag tag (Fig. 1). All complemented strains proved to be sufficiently viable (see below, and data not shown) to allow their in-depth analysis. Our strain sets included strains with Pf Hsp90, yeast Hsp82, human $\mathrm{Hsp} 90 \alpha$, and human Hsp90 $\beta$. It is noteworthy that a continued auxotrophic selection for maintenance of the episomes was not necessary since the expression of an Hsp90 is essential for viability. Hence, the HSP90 genes served themselves as a marker and all strains could be grown in rich medium.

We proceeded to determine the relative levels of the different Hsp90 proteins with an immunoblot experiment. Total cell extracts were prepared from all strains and probed with a monoclonal antibody against the Flag tag (Fig. 2A). An extract from a strain with untagged human Hsp90 $\beta$ (lane $\beta$ ) served as a negative control. By comparison with the total cellular proteins revealed on the filter by Ponceau S-staining, it is apparent that the levels of the four Flag-tagged Hsp90 proteins are overall quite comparable, human Hsp90 $\alpha$ being the lowest with a 2 -fold difference compared to yeast Hsp82. When a similar immunoblot was probed with antibodies that are specific for yeast Hsp90, no signal could be detected in any of the strains complemented with human or Pf Hsp90. The strain expressing Flag-tagged yeast Hsp82 served as a positive control. This result ascertained that the strains $F \alpha, F \beta$ and FP expressing Flag-tagged human Hsp90 $\alpha$, Hsp90 $\beta$ and Pf Hsp90, respectively, indeed lack the endogenous yeast HSP90 genes. 

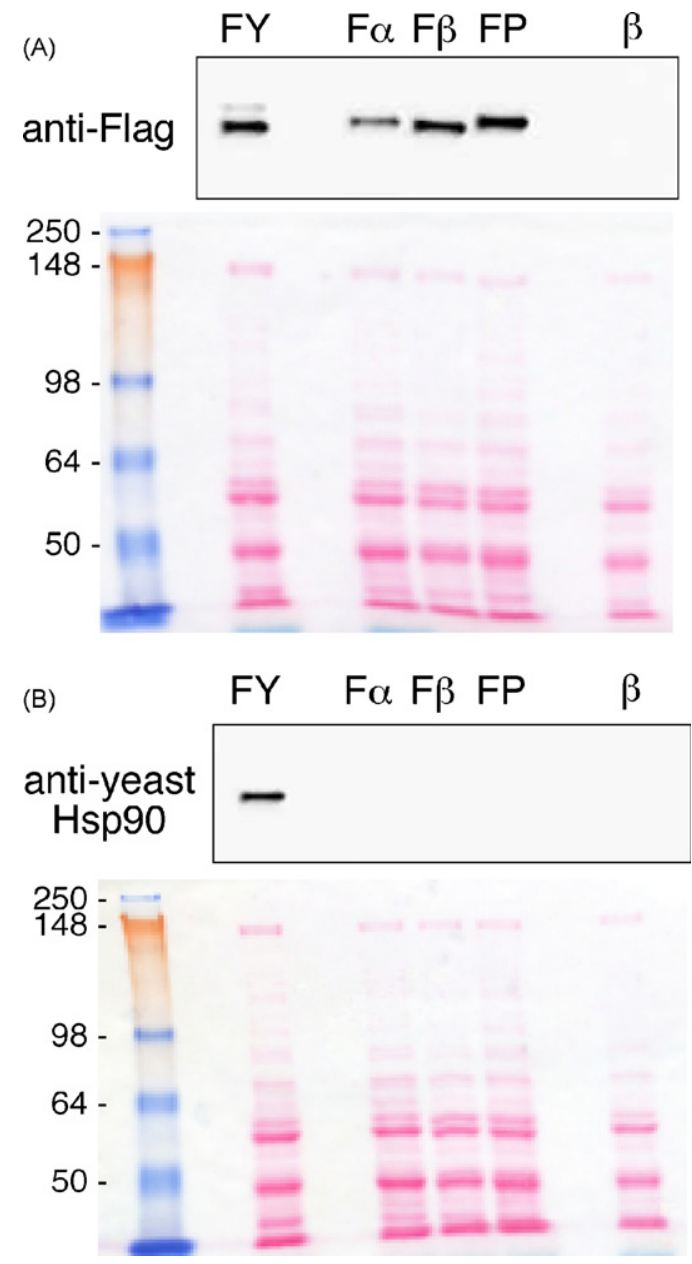

Fig. 2. Immunoblot analysis of Hsp90 proteins. Equal amounts of extracts from indicated strains were probed with a monoclonal against the Flag tag (panel A) or antibodies against yeast Hsp90 (panel B). The leftmost lanes on the Ponceau Sstained filters shown below the immunoblots in both panels are molecular weight markers (sizes indicated in $\mathrm{kDa}$ ). The relative band intensities of the Flag-tagged Hsp90 proteins are $100 \%, 49 \%, 99 \%$ and $125 \%$ for FY, F $\alpha, F \beta$ and FP, respectively.

We had previously compared the growth of cells with tagged or untagged yeast Hsp82 (data not shown) and had not noticed any particular difference. Since pilot experiments indicated that FP grew less well than FY (see below), we wanted to determine whether the Flag tag might adversely affect complementation by Pf Hsp90. Serial dilutions of equal numbers of cells of the two corresponding strains, P and Pf (with untagged and tagged Pf Hsp90, respectively), were spotted onto solid medium (Fig. 3A). It became clear that the Flag tag, if anything, slightly improved the function (or expression) of Pf Hsp90.

Thus, having established that Hsp90 protein levels were not too dissimilar and Flag-tagged versions of Hsp90 appropriate, we could start testing our set of strains consisting of the four Flagtagged Hsp90 proteins. On plates, FP and F $\alpha$ turned out to grow considerably more slowly than FY and F $\beta$ although they eventually reached similar colony numbers and sizes. We realized that, if we were going to use plate growth assays to assess the effects of Hsp90 inhibitors, we would need more comparable cell densities. We therefore chose to spot FP and F $\alpha 1$ day earlier than FY and $\mathrm{F} \beta$. With this 1 day advance, all four strains grow to comparable densities within an additional 4 days, FY being yet slightly more vigorous (Fig. 3B). To obtain more quantitative data, growth assays were repeated in liquid culture (Fig. $3 C$ ). In this case, all strains were subjected to identical conditions. These experiments confirmed the slow and vigorous growth of FP and FY, respectively. Ultimately, all four strains reach similar saturating densities. Surprisingly, in liquid culture the yeast strain with human $\mathrm{Hsp} 90 \alpha(\mathrm{F} \alpha)$ does not display the prominent growth defect that is obvious on plates and grows even slightly faster than $\mathrm{F} \beta$.

Next, we explored whether plate or liquid growth assays would allow us to test Hsp90 inhibitors and to identify differences between these tester strains. After pilot experiments with a range of inhibitor concentrations, we settled on concentrations that gave the largest differences without completely obliterating growth of all strains except the comparatively more resistant FY (data not shown). The result of a growth assay on plates is shown in Fig. 4. It must be pointed out again that F $\alpha$ and FP were spotted 1 day earlier. However, this was the case for all treatments and, therefore, their relative sensitivities to the drugs can be compared. While FY is only slightly affected by either $40 \mu \mathrm{M}$ GA or $2 \mu \mathrm{M}$ Rd, FP is exquisitely sensitive to GA and to a lesser extent to $2 \mu \mathrm{M}$ Rd. The strains with human Hsp90 isoforms ( $F \alpha$ and $F \beta$ ) are similarly affected and more resistant to GA than FP. FP in turn is more resistant to Rd than both F $\alpha$ and $\mathrm{F} \beta$. As could be expected from the results discussed above, the four strains behaved slightly differently in liquid medium (Fig. 5). Although the differences in Rd sensitivities between human ( $\mathrm{F} \alpha$ and

(A)
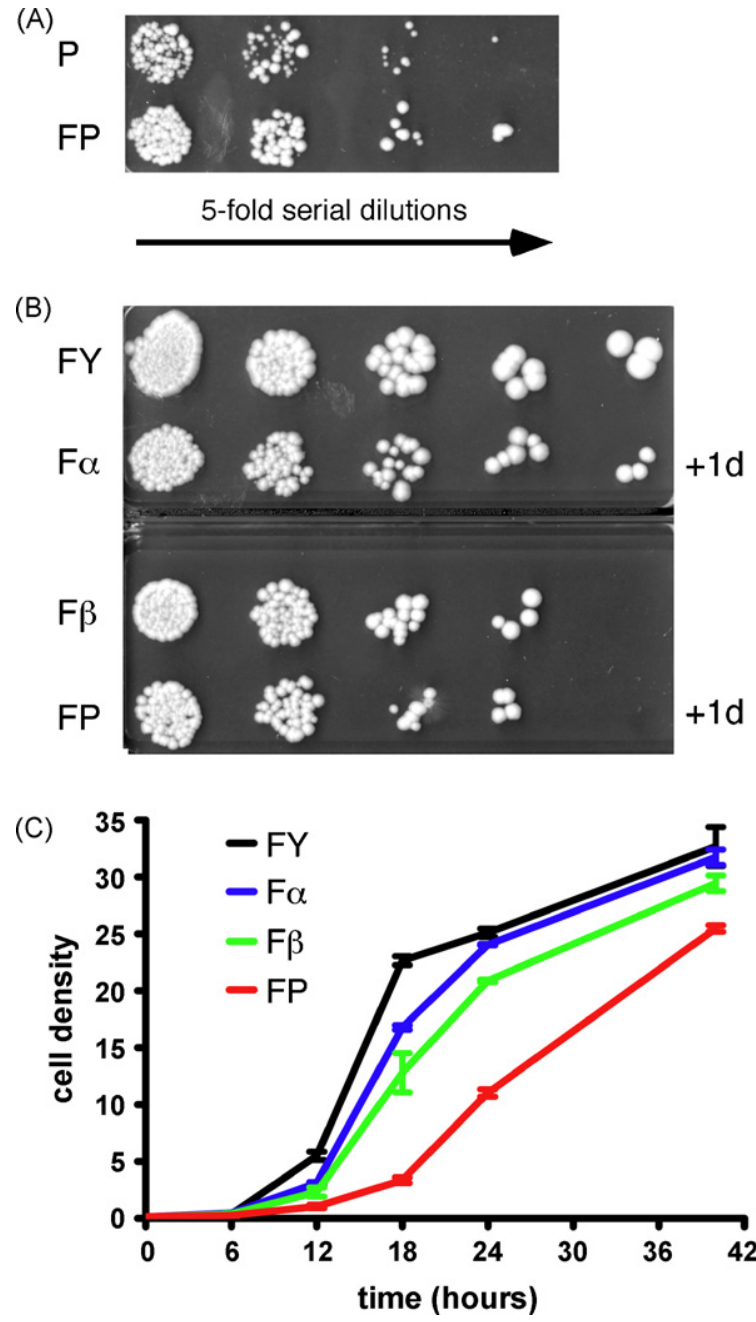

Fig. 3. Growth assays with Hsp90 tester strains in the absence of Hsp90 inhibitors (A) Comparison of strains with untagged and Flag-tagged $\mathrm{Pf} \mathrm{Hsp} 90$ ( $\mathrm{P}$ and FP, respectively) with a plate growth assay. (B) Plate growth assay with all four Hsp90 tester strains. F $\alpha$ and FP were spotted 1 day earlier (hence the indication $+1 \mathrm{~d}$ for one additional day) and the image was acquired on day 4 (relative to when FY and F $\beta$ were spotted). (C) Growth curves determined with liquid cultures. Cell density refers to OD600 values. Error bars indicate the standard deviation. 


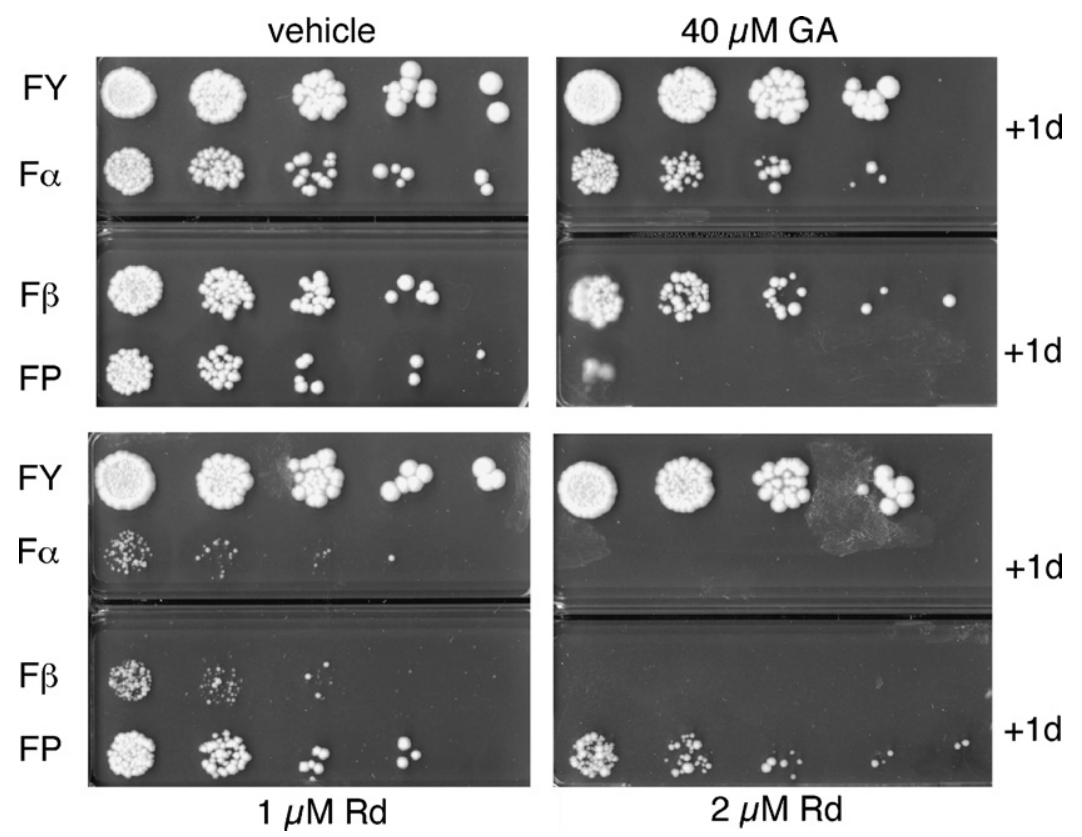

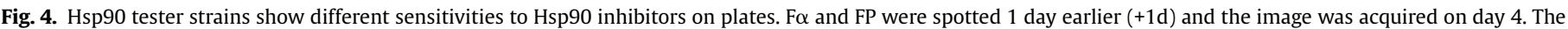
solvent DMSO (vehicle) was added as indicated in Section 2.

F $\beta$ ) and Pf Hsp90 (FP) strains disappeared, GA was still more toxic to FP. To reveal the differential sensitivities of the four strains to the two drugs more clearly, cell densities in the presence of the drugs were standardized to those of untreated cultures and expressed as a ratio (Fig. 5D). FY, the strain that grows the most rapidly and is only slightly affected by either drug, is relatively more inhibited by GA for the first $18 \mathrm{~h}$. In the presence of GA, it reaches saturation about $6 \mathrm{~h}$ later, but it does reach it, which is why the ratio drops back down to 1 at $24 \mathrm{~h}$. Most importantly, this plot shows unambiguously that FP is hypersensitive to GA compared to Rd and that in that respect it differs substantially from the strains with human Hsp90 ( $\mathrm{F} \alpha$ and $\mathrm{F} \beta$ ). Moreover, this quantitative result agrees with the qualitative one from the plate assays, which already indicated that FP is more sensitive to GA than Rd and hypersensitive to GA by comparison with strains expressing human Hsp90.

\section{Discussion}

The number of known Hsp90 substrates is continuing to grow very rapidly (for an updated list, see http://www.picard.ch/downloads/Hsp90interactors.pdf) as could be expected for a molecular chaperone that may be the most abundant cytosolic protein even in unstressed cells [24]. It is still difficult to provide a comprehensive list of the substrates that account for the essential functions of Hsp90 for viability of both unicellular and metazoan organisms. We now add Pf Hsp90 to the list of heterologous Hsp90's that can apparently fulfill at least the essential functions of Hsp90 in yeast for vegetative growth in standard growth media. Whatever these functions are exactly, they must include many of the ones that have been highlighted by global biochemical and genetic analyses of Hsp90 interactions in this organism [25-28]. The results of these genetic complementation experiments underscore the high evolutionary conservation of Hsp90 [29-32]. For example, yeast Hsp82 compared to human Hsp90 $\alpha$ and Pf Hsp90 is 59\% and 58\% identical, respectively. The sequence identity drops to only $36 \%$ with the Escherichia coli Hsp90 ortholog htpG, which may explain why htpG fails to complement yeast ([16], G. Palmer and DP, unpublished results). Despite the remarkable complementation by heterologous Hsp90's for viabil- ity, this complementation is far from complete. In the absence of the Hsp90 co-chaperone Sti1, C. elegans Hsp90 and human Hsp90 $\beta$ cannot support viability of yeast mutants [17]. Moreover, human Hsp90 $\beta$ fails to support Ste11-dependent pheromone signaling in yeast [19], and yeast and human Hsp90's differ in their ability to support several other endogenous and exogenous substrates [33]. These shortcomings are mirrored by our findings that human and Pf Hsp90's do not support wild-type growth rates (Figs. 3-5). This is particularly evident for the strain with Pf Hsp90, which grows substantially more slowly than our control yeast strain with Hsp82.

It is noteworthy that the relative growth rates also differ between liquid and solid media. It is a well-known phenomenon in $S$. cerevisiae that the genetic background can differentially affect the ability of a strain to grow in a plate assay or in liquid culture (see for example refs. [34-36]). With respect to Hsp90, our findings may indicate that different environmental conditions impose subtly different Hsp90 substrate requirements, which are differentially fulfilled by different Hsp90 proteins. While our results demonstrate that heterologous Hsp90's do not behave differently because of vastly different expression levels, there are several other parameters that can be considered. They might differ in their ability to bind and to cooperate with the yeast Hsp90 co-chaperones or to process yeast substrates (see also afore-mentioned examples). Alternatively, they might not function optimally because of differences in posttranslational modifications or because of the particulars of the chemical milieu of a yeast cell.

We found that all strains of the Hsp90 tester set are sensitive to Hsp90 inhibitors, albeit to very different extents. Strain FY with the yeast Hsp82 was the least sensitive one and the only one that could eventually reach similar cell densities with drugs as without drugs both on plates and in liquid culture. Higher concentrations yet are necessary to inhibit growth of FY more strongly (data not shown), in agreement with previous reports about the toxicity of Hsp90 inhibitors to wild-type strains, notably in a $\Delta p d r 5$ background $[22,37]$. At concentrations that were only minimally toxic to the strain FY, the other three strains were severely growth impaired. Surprisingly, Piper and co-workers [33] have reported that a strain with human Hsp90 $\alpha$ displayed relative resistance to Rd compared to strains with human Hsp90ß or yeast Hsc82 or Hsp82. This discrepancy could be due to any of a number of differences in the 

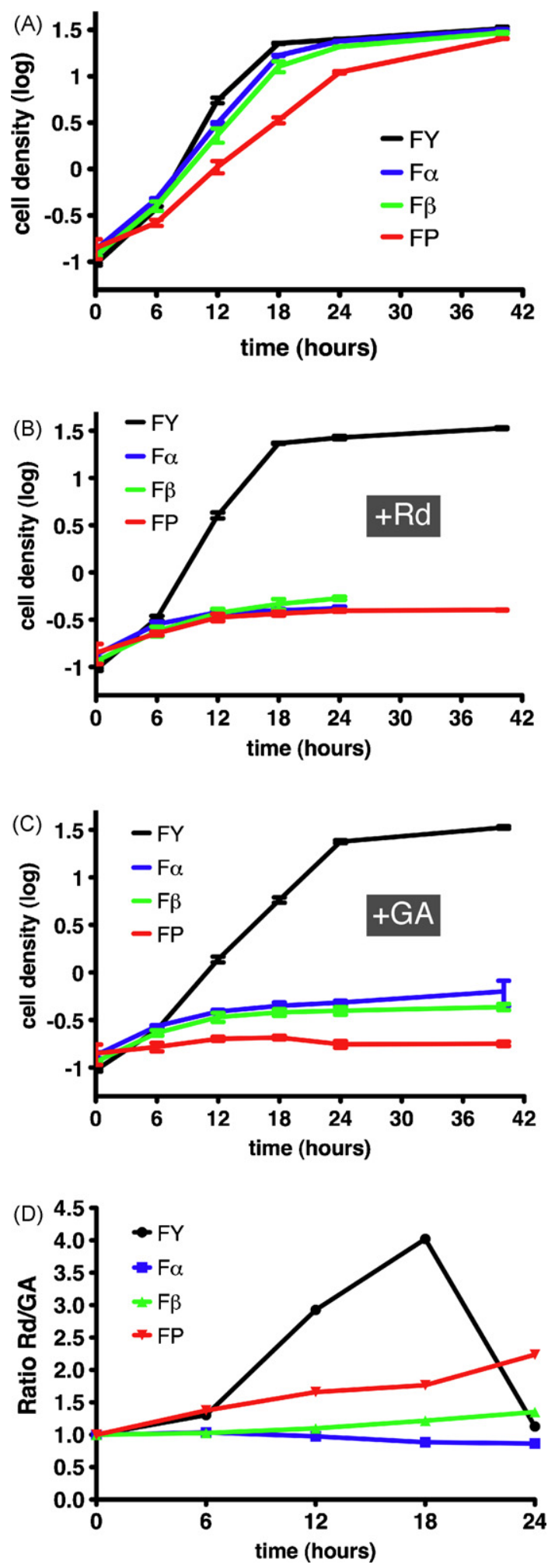

Fig. 5. Hsp90 tester strains show different sensitivities to Hsp90 inhibitors in liquid culture. Panels A, B and C show growth curves of cultures that were left untreated (only solvent), treated with $2 \mu \mathrm{M}$ Rd and $40 \mu \mathrm{M}$ GA, respectively. The scale of the $Y$-axes is logarithmic. Error bars indicate the standard deviation. The data plotted in panel $\mathrm{A}$ is the same as that of panel $\mathrm{C}$ in Fig. 3, but here the scale of the $Y$-axis is different. (D) This graph shows the ratios of standardized cell densities observed with the two inhibitors. Note that the last time point of panels $\mathrm{A}-\mathrm{C}$ is not computed here and that the scale of the $Y$-axis is linear. experimental set-ups. Different expression levels of the drug target, Hsp90, could easily explain differences in drug sensitivity. While we do not know whether Hsp90 $\alpha$ and Hsp90 $\beta$ levels were similar in the afore-mentioned study, we can assert for our strains that they differed by at most 2-fold (Fig. 2) because the two isoforms were marked by the same epitope tag and thus recognized by the same antibody with identical efficiency. Most notably, the levels of human Hsp90 $\beta$ and Pf Hsp90 were very close to those of the isogenic strain with yeast Hsp82.

Hsp90 inhibitor sensitivity is determined by a variety of parameters including cell permeability, export pumps, metabolism, and the binding affinity for Hsp90. Undoubtedly, the latter is the most relevant in the context of the differential sensitivities of different strains that only differ by the Hsp90 proteins they express. They may have intrinsically different binding affinities for one drug or another. Whether that is the case for yeast, human and Pf Hsp90 is not known since, to the best of our knowledge, they have not yet been measured side by side. Furthermore, the binding affinities of Hsp90 for inhibitors can be influenced by its interactions with co-chaperones [17,22,37-41], which in turn may be modulated by posttranslational modifications of Hsp90 (see for example refs. [42-44]). Since we had found that the Hsp90 co-chaperone p23 directly protects Hsp90 from binding inhibitors [22], we did introduce the Pf p23 ortholog into yeast. Instead of improving Pf Hsp90 function and resistance, expression of Pf p23 in FP further compromised growth (data not shown). To reconstitute Pf Hsp90 function fully in yeast, it may be necessary to introduce the entire or at least more of the Pf co-chaperone complement.

Whatever the reasons are for the observed differential sensitivities, we believe that our results constitute a proof of principle that it should be possible to use this set of yeast strains to screen for Hsp90 inhibitors with an even larger differential between the Hsp90 of the host and the Hsp90 of the deadly parasite. Moreover, the Hsp90 set could easily be expanded to include Hsp90 from other unicellular parasites such as Trypanosomes and Leishmania. In closing, we would like to emphasize that this is a powerful live cell, but nevertheless artificial, screening tool. The efficiency and parasite-specificity of candidate drugs will need to be ascertained and further characterized in appropriate in vitro and in vivo systems with the parasite itself.

\section{Acknowledgments}

We are grateful to Drs. Peter Piper, Natasha Kralli, and Françoise Stutz for providing strains and plasmids. We thank Fedor Forafonov for the construction of plasmid pHGF.

\section{References}

[1] Picard D. Heat-shock protein 90, a chaperone for folding and regulation. Cell Mol Life Sci 2002;59:1640-8.

[2] Neckers L. Heat shock protein 90: the cancer chaperone. J Biosci 2007;32:517-30

[3] Pearl LH, Prodromou C, Workman P. The Hsp90 molecular chaperone: an open and shut case for treatment. Biochem J 2008;410:439-53.

[4] Tanaka Y, Shiomi K, Kamei K, et al. Antimalarial activity of radicicol, heptelidic acid and other fungal metabolites. J Antibiot 1998;51:153-60.

[5] Banumathy G, Singh V, Pavithra SR, Tatu U. Heat shock protein 90 function is essential for Plasmodium falciparum growth in human erythrocytes. J Biol Chem 2003;278:18336-45.

[6] Kumar R, Musiyenko A, Barik S. The heat shock protein 90 of Plasmodium falciparum and antimalarial activity of its inhibitor, geldanamycin. Malar J 2003;2:30.

[7] Pavithra SR, Kumar R, Tatu U. Systems analysis of chaperone networks in the malarial parasite Plasmodium falciparum. PLoS Comput Biol 2007;3:1701-15.

[8] Whitesell L, Lindquist SL. HSP90 and the chaperoning of cancer. Nat Rev Cancer 2005;5:761-72.

[9] Workman P, Burrows F, Neckers L, Rosen N. Drugging the cancer chaperone HSP90: combinatorial therapeutic exploitation of oncogene addiction and tumor stress. Ann N Y Acad Sci 2007;1113:202-16. 
[10] Geller R, Vignuzzi M, Anion R, Frydman J. Evolutionary constraints on chaperone-mediated folding provide an antiviral approach refractory to development of drug resistance. Genes Dev 2007;21:195-205.

[11] Kumar R, Pavithra SR, Tatu U. Three-dimensional structure of heat shock protein 90 from Plasmodium falciparum: molecular modelling approach to rational drug design against malaria. J Biosci 2007;32:531-6.

[12] Borkovich KA, Farrelly FW, Finkelstein DB, Taulien J, Lindquist S. Hsp82 is an essential protein that is required in higher concentrations for growth of cells at higher temperatures. Mol Cell Biol 1989;9:3919-30.

[13] Minami Y, Kimura Y, Kawasaki H, Suzuki K, Yahara I. The carboxy-terminal region of mammalian HSP90 is required for its dimerization and function in vivo. Mol Cell Biol 1994;14:1459-64.

[14] Picard D, Khursheed B, Garabedian MJ, Fortin MG, Lindquist S, et al., Yamamoto KR. Reduced levels of hsp90 compromise steroid receptor action in vivo. Nature 1990;348:166-8.

[15] Palmer G, Louvion J-F, Tibbetts RS, Engman DM, Picard D. Trypanosoma cruzi heat-shock protein 90 can functionally complement yeast. Mol Biochem Parasitol 1995;70:199-202.

[16] Yue L, Karr TL, Nathan DF, Swift H, Srinivasan S, Lindquist S. Genetic analysis of viable Hsp90 alleles reveals a critical role in Drosophila spermatogenesis. Genetics 1999;151:1065-79.

[17] Piper PW, Panaretou B, Millson SH, et al. Yeast is selectively hypersensitised to heat shock protein 90 (Hsp90)-targeting drugs with heterologous expression of the human Hsp90 $\beta$, a property that can be exploited in screens for new Hsp90 chaperone inhibitors. Gene 2003;302:165-70.

[18] Sikorski RS, Hieter P. A system of shuttle vectors and yeast host strains designed for efficient manipulation of DNA in Saccharomyces cerevisiae. Genetics 1989;122:19-27.

[19] Louvion J-F, Abbas-Terki T, Picard D. Hsp90 is required for pheromone signaling in yeast. Mol Biol Cell 1998;9:3071-83.

[20] MacLean MJ, Martínez Llordella M, Bot N, Picard D. A yeast-based assay reveals a functional defect of the Q488H polymorphism in human Hsp90 $\alpha$. Biochem Biophys Res Commun 2005;337:133-7.

[21] Panaretou B, Prodromou C, Roe SM, et al. ATP binding and hydrolysis are essential to the function of the Hsp90 molecular chaperone in vivo. EMBO J 1998; 17:4829-36.

[22] Forafonov F, Toogun OA Grad I, Suslova E, Freeman BC, Picard D. p23/Sba1p protects against Hsp90 inhibitors independently of its intrinsic chaperone activity. Mol Cell Biol 2008;28:3446-56.

[23] Gueldener U, Heinisch J, Koehler GJ, Voss D, Hegemann JH. A second set of loxP marker cassettes for Cre-mediated multiple gene knockouts in budding yeast. Nucleic Acids Res 2002;30:e23.

[24] Lai B-T, Chin NW, Stanek AE, Keh W, Lanks KW. Quantitation and intracellular localization of the $85 \mathrm{~K}$ heat shock protein by using monoclonal and polyclonal antibodies. Mol Cell Biol 1984;4:2802-10.

[25] Zhao R, Davey M, Hsu YC, et al. Navigating the chaperone network: integrative map of physical and genetic interactions mediated by the Hsp90 chaperone. Cell 2005;120:715-27.

[26] Millson SH, Truman AW, King V, Prodromou C, Pearl LH, Piper PW. A two-hybrid screen of the yeast proteome for hsp90 interactors uncovers a novel Hsp90 chaperone requirement in the activity of a stress-activated mitogen-activated protein kinase, Slt2p (Mpk1p). Eukaryot Cell 2005;4:849-60.
[27] Albanèse V, Yam AY, Baughman J, Parnot C, Frydman J. Systems analyses reveal two chaperone networks with distinct functions in eukaryotic cells. Cell 2006;124:75-88.

[28] McClellan AJ, Xia Y, Deutschbauer AM, Davis RW, Gerstein M, Frydman J. Diverse cellular functions of the Hsp90 molecular chaperone uncovered using systems approaches. Cell 2007;131:121-35.

[29] Emelyanov VV. Phylogenetic relationships of organellar Hsp90 homologs reveal fundamental differences to organellar Hsp70 and Hsp60 evolution. Gene 2002;299:125-33.

[30] Stechmann A, Cavalier-Smith T. Phylogenetic analysis of eukaryotes using heatshock protein Hsp90. J Mol Evol 2003;57:408-19.

[31] Stechmann A, Cavalier-Smith T. Evolutionary origins of Hsp90 chaperones and a deep paralogy in their bacterial ancestors. J Eukaryot Microbiol 2004;51:364-73.

[32] Chen B, Zhong D, Monteiro A. Comparative genomics and evolution of the HSP90 family of genes across all kingdoms of organisms. BMC Genomics 2006;7: 156.

[33] Millson SH, Truman AW, Rácz A, et al. Expressed as the sole Hsp90 of yeast, the $\alpha$ and $\beta$ isoforms of human Hsp90 differ with regard to their capacities for activation of certain client proteins, whereas only Hsp90 $\beta$ generates sensitivity to the Hsp90 inhibitor radicicol. FEBS J 2007;274:4453-63.

[34] McCusker JH, Haber JE. Mutations in Saccharomyces cerevisiae which confer resistance to several amino acid analogs. Mol Cell Biol 1990;10:2941-9.

[35] Codon AC, Gasent-Ramirez JM, Benitez T. Factors which affect the frequency of sporulation and tetrad formation in Saccharomyces cerevisiae baker's yeasts. Appl Environ Microbiol 1995;61:630-8.

[36] Abruzzi K, Denome S, Olsen JR, et al. A novel plasmid-based microarray screen identifies suppressors of rrp6 $\Delta$ in Saccharomyces cerevisiae. Mol Cell Biol 2007;27:1044-55.

[37] Piper PW, Millson SH, Mollapour M, et al. Sensitivity to Hsp90-targeting drugs can arise with mutation to the Hsp90 chaperone, cochaperones and plasma membrane ATP binding cassette transporters of yeast. Eur J Biochem 2003;270:4689-95.

[38] Bandhakavi S, McCann RO, Hanna DE, Glover CV. A positive feedback loop between protein kinase CKII and Cdc37 promotes the activity of multiple protein kinases. J Biol Chem 2003;278:2829-36.

[39] Dolinski KJ, Cardenas ME, Heitman J. CNS1 encodes an essential p60/Sti1 homolog in Saccharomyces cerevisiae that suppresses cyclophilin 40 mutations and interacts with Hsp90. Mol Cell Biol 1998;18:7344-52.

[40] Song Y, Masison DC. Independent regulation of Hsp70 and Hsp90 chaperones by Hsp70/Hsp90-organizing protein Sti1 (Hop1). J Biol Chem 2005;280:34178-85.

[41] Holmes JL, Sharp SY, Hobbs S, Workman P. Silencing of HSP90 cochaperone AHA1 expression decreases client protein activation and increases cellular sensitivity to the HSP90 inhibitor 17-allylamino-17-demethoxygeldanamycin. Cancer Res 2008;68:1188-97.

[42] Kovacs JJ, Murphy PJ, Gaillard S, et al. HDAC6 regulates Hsp90 acetylation and chaperone-dependent activation of glucocorticoid receptor. Mol Cell 2005;18:601-7.

[43] Scroggins BT, Robzyk K, Wang D, et al. An acetylation site in the middle domain of Hsp90 regulates chaperone function. Mol Cell 2007;25:151-9.

[44] Yang Y, Rao R, Shen J, et al. Role of acetylation and extracellular location of heat shock protein $90 \alpha$ in tumor cell invasion. Cancer Res 2008;68:4833-42. 